\title{
Influence of the Lower Ionosphere on Propagation of VLF Waves to Great Distances
}

\author{
James R. Wait
}

Contribution from Central Radio Propagation Laboratory, National Bureau of Standards Boulder, Colo.

(Received February 28, 1963)

\begin{abstract}
Theoretical attenuation and phase characteristics at VLF are presented for a number of idealized models of the lower ionosphere. The results indicate the limitations of the sharply bounded model.
\end{abstract}

\section{Introduction}

Despite the great number of papers dealing with VLF radio propagation, there appears to be a need to consolidate some of the recent theoretical data. It is the purpose of this paper to fulfill this need. The intent is to present results, in convenient graphical form, which illustrate the expected value of the attenuation and phase velocity of the dominant modes in the frequency range from 8 to $30 \mathrm{kc} / \mathrm{s}$. Some of the effects considered are due to the earth curvature, ground conductivity, and finally the gradient of the effective conductivity of the lower ionosphere.

No attempt will be made here to describe the mathematical aspects of the subject in any detail. However, certain basic formulas will be introduced to permit comparisons with previous work [Wait, $1962]$.

The basic theoretical model is a smooth spherical earth of homogeneous conductivity surrounded by a concentric (spherically) stratified ionosphere. Initially the ionosphere is regarded as a sharply bounded isotropic medium. For this discussion the influence of the terrestrial magnetic field is not considered. The non-sharpness or diffuseness of the lower edge of the ionosphere is treated by using an exponential isotropic model which has the virtue of simplicity.

\section{Basic Modal Equation}

To permit a straightforward treatment, it is assumed that the level $h$ above the ground may be characterized by an ionospheric reflection coefficient $R_{i}$. Furthermore, it is convenient to make the substitution

$$
R_{i}=-\exp \left(\alpha C^{\prime}\right),
$$

where $C^{\prime}$ is the cosine of the angle of incidence at the reference level in the ionosphere. While the $\alpha$ is generally a (complex) function of $C^{\prime}$, it is found that under most practical situations $\alpha$ is approximately a constant for $C^{\prime}$ ranging from 0 up to about 0.3 .
This, of course, encompasses the important angles in long distance propagation of VLF radio waves.

The relevant modal equation for the low order (important) TM modes may now be written [Wait, 1962]

$$
\begin{aligned}
\frac{2}{3}(k a)\left(C^{2}+\frac{2 h}{a}\right)^{3 / 2}+ & i \alpha\left(C^{2}+\frac{2 h}{a}\right)^{1 / 2} \\
& +i \log \frac{w_{2}^{\prime}(t)}{w_{1}^{\prime}(t)}-(4 n-1) \frac{\pi}{2}=0,
\end{aligned}
$$

where

$$
\begin{aligned}
C^{2} & =\left(C^{\prime}\right)^{2}-\frac{2 h}{a}, \\
k & =2 \pi / \text { wavelength, } \\
a & =\text { radius of the earth, } \\
n & =\text { mode number, }
\end{aligned}
$$

and

$$
t=-(k a / 2)^{2 / 3} C^{2} \text {. }
$$

This form of the mode equation is valid for a ground surface which is effectively a perfect conductor. The logarithmic term involves the derivatives of the Airy functions of argument $t$.

Under the somewhat unrealistic assumptions that the ionosphere is a sharply bounded ionized medium and the terrestrial magnetic field may be neglected, it is known that [Wait, 1962]

$$
\alpha \cong-2\left(\frac{i \omega}{\omega_{r}}\right)^{1 / 2}\left(1+\frac{\omega_{r}}{i w}\right),
$$

where $\omega_{r}=\omega_{o}^{2} / \nu . \quad$ Here

$$
\begin{aligned}
\omega_{0} & =\text { the (angular) plasma frequency, } \\
\nu & =\text { the effective collision frequency. }
\end{aligned}
$$
by

The attenuation $A_{n}$, of the $n$th mode, is then given

$$
A_{n}=-\operatorname{Im} k S_{n} \text { nepers/meter, }
$$

where $S_{n}=\left(1-C_{n}^{2}\right)^{\frac{1}{2}} \cong 1-C_{n}^{2} / 2$. In more convenient units 


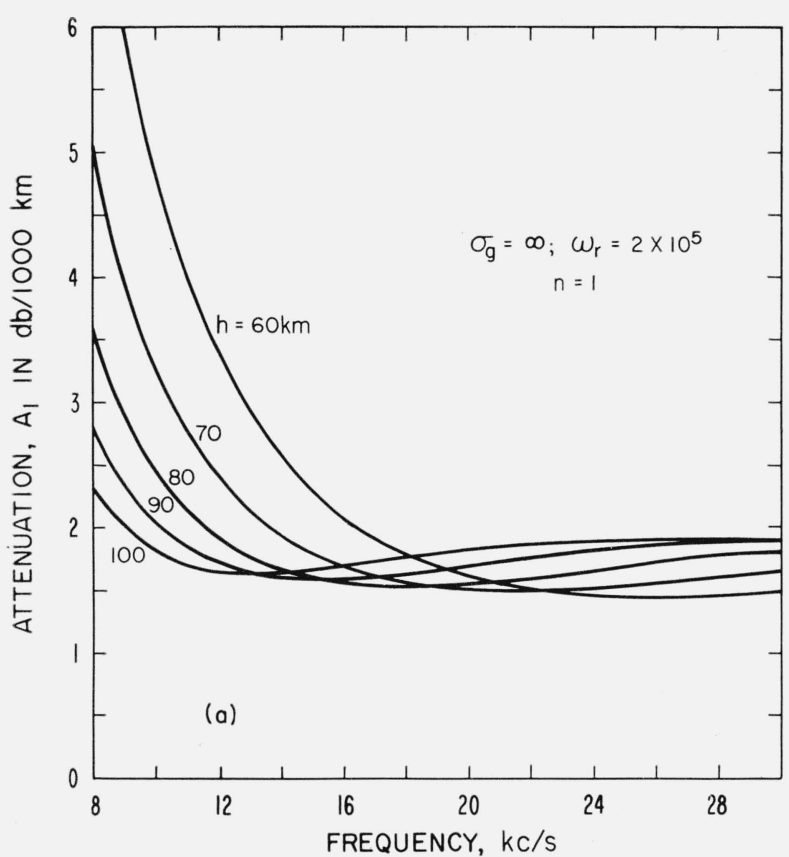

FIgURE 1a. Attenuation rate of the first mode for a perferctly conducting earth and a sharply bounded ionosphere.

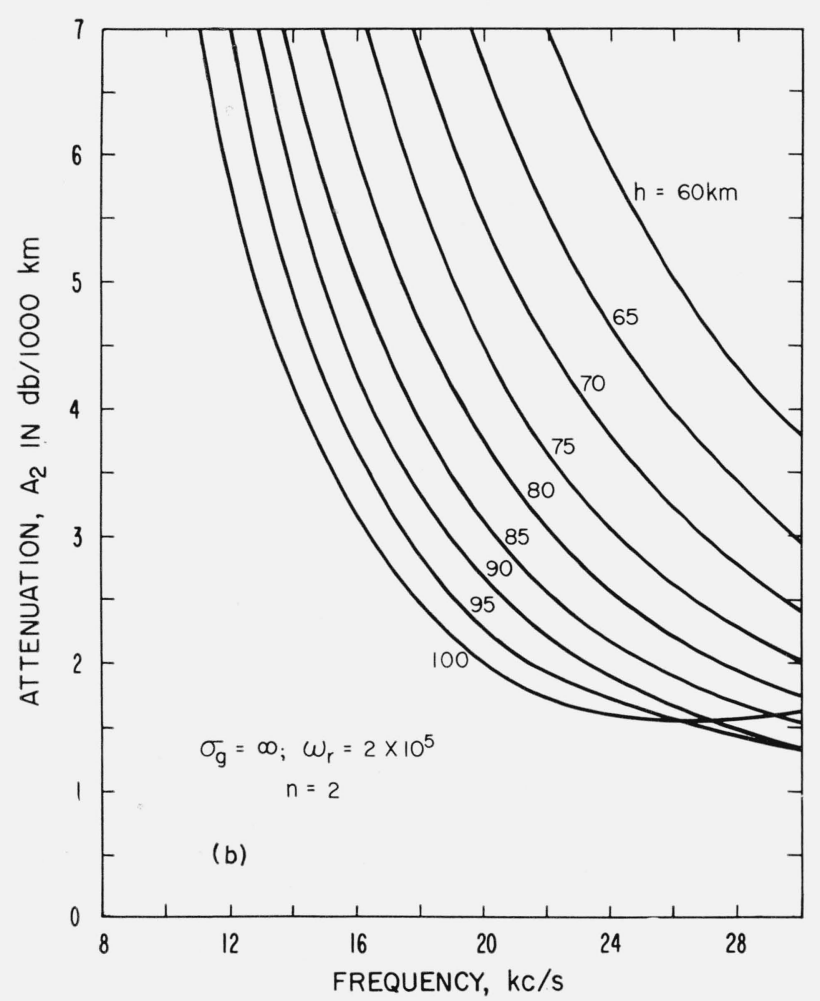

FIGURE 1b. Attenuation rate of the second mode for a perfectly conducting earth and a sharply bounded ionosphere.
$A_{n} \cong \operatorname{Im} k C_{n}^{2} / 2 \times 8.68 \times 10^{3}$ in $\mathrm{db} / 1000 \mathrm{~km}$,

when

$$
k=2 \pi f_{\mathrm{kc} / \mathrm{s}} / 300,
$$

where $f_{\mathrm{ke} / \mathrm{s}}$ is the frequency in kilocycles per second.

The phase velocity $v_{n}$ of the $n$th mode, referred to the velocity of light in vacuo, is then obtained from

$$
\frac{v_{n}}{c}-1 \cong \operatorname{Re}\left(C_{n}^{2} / 2\right)
$$

The complex values of $C_{n}$ are the roots of the modal equation quoted above. It is considered to be convenient to number the modes in the VLF range so that mode number 1 is the mode of least attenuation. Higher order modes correspond to modes of progressively greater attenuation.

\section{Attentuation for the Sharply Bounded Model}

Using (2) and (4), the attenuation rates $A_{1}$ and $A_{2}$ for the first two modes of lowest attenuation are shown in figures $1 \mathrm{a}$ and $1 \mathrm{~b}$, respectively. The height of the reflecting layer varies from 60 to $100 \mathrm{~km}$ in 5 or $10 \mathrm{~km}$ intervals. The ground conductivity $\sigma_{g}$ is here taken to be $\infty$ while $\omega_{r}$ has the value $2 \times 10 .^{5}$ The latter is typical of daytime conditions. It is interesting to note that the attenuation $A_{1}$ for the first mode has a broad minimum when plotted as a function of frequency. It is also noted that at the lower frequencies the attenuation $A_{1}$ increases rather significantly for the lower reflecting heights.

In general it is indicated that the attenuation $A_{2}$ of the second mode is somewhat greater than $A_{1}$. It is important to observe that $A_{2}$ is very dependent on the height parameter $h$.

The influence of finite ground conductivity can be determined by solving a slightly more complicated form of the modal equation. To within a good approximation it has the same form as (2) if the log term is replaced by

$$
i \log \left[\frac{w_{2}^{\prime}(t)-q w_{2}(t)}{w_{1}^{\prime}(t)-q w_{1}(t)}\right]
$$

where

$$
q=-i(k a / 2)^{\frac{1}{3}} Z_{g} / 120 \pi,
$$

and where $Z_{g}$ is the surface impedance of the ground. Choosing the ground conductivity $\sigma_{g}$ to have a number of typical values, $A_{1}$ was calculated from the modified form of the mode equation. It appears that for propagation over sea water (i.e., $\sigma_{g} \simeq 4000$ millimhos $/ \mathrm{m}$ ) the value of $\sigma_{g}$ is effectively infinite. However, over moderately or poorly conducting land, the effect of finite ground conductivity cannot be ignored as indicated clearly in figure 1c. 


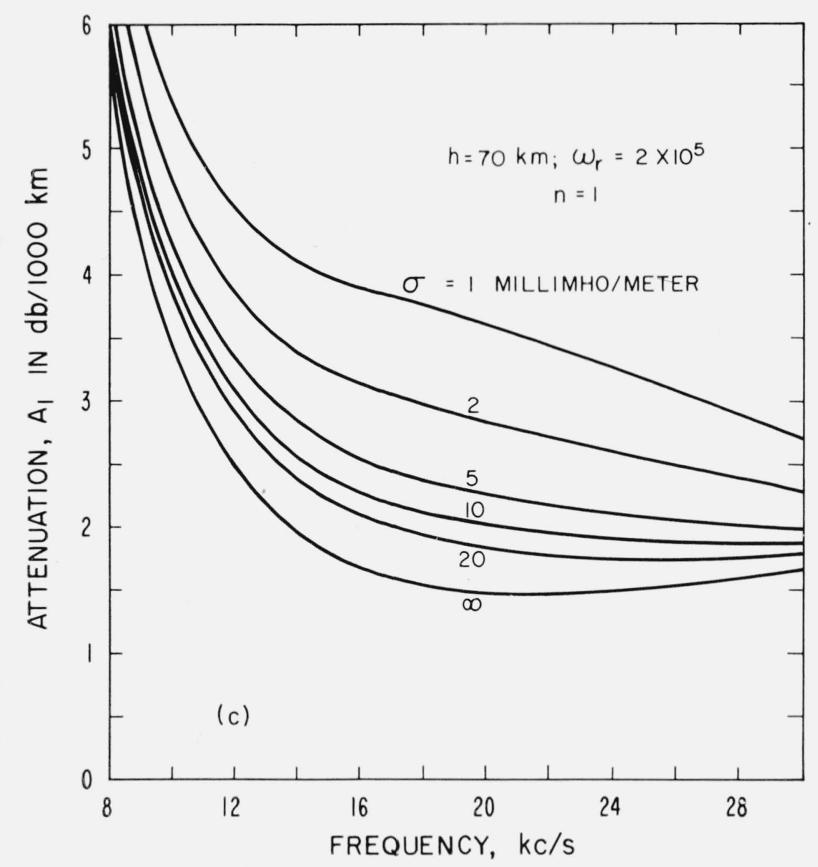

FIGURE 1c. Attenuation rate of the first mode for an imperfectly conducting earth and a sharply bounded ionosphere.

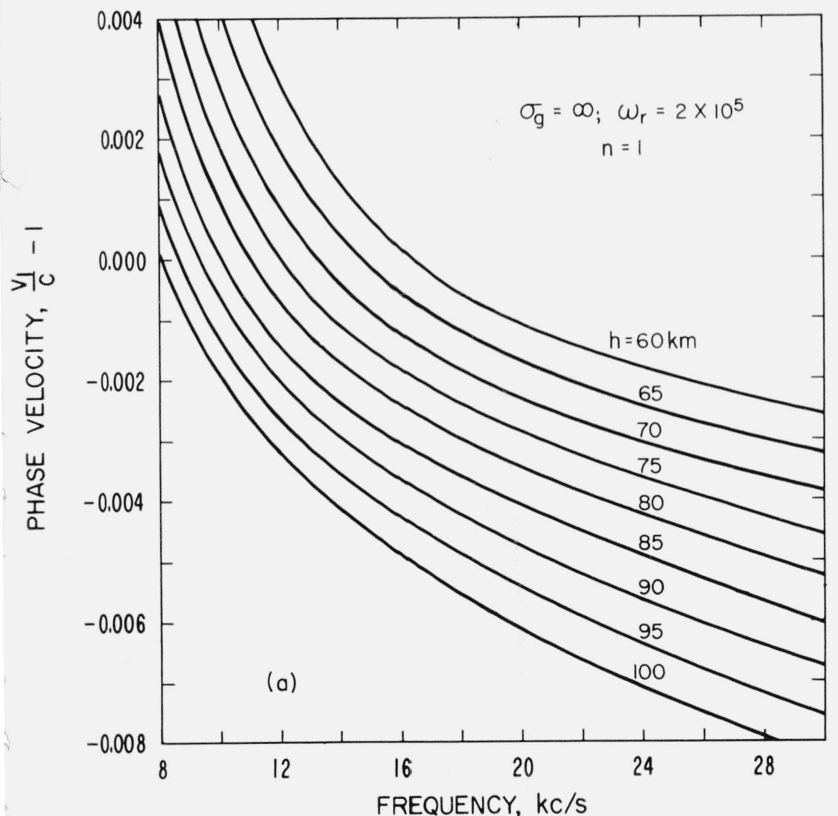

Figure 2a. Phase velocity of the first mode for a perfectly conducting earth and a sharply bounded ionosphere.

\section{Phase Velocity for the Sharply Bounded Model}

The values of the phase velocity of the modes 1 and 2 for the same conditions as in section 3 are shown in figures $2 \mathrm{a}, 2 \mathrm{~b}$, and $2 \mathrm{c}$. As expected, the

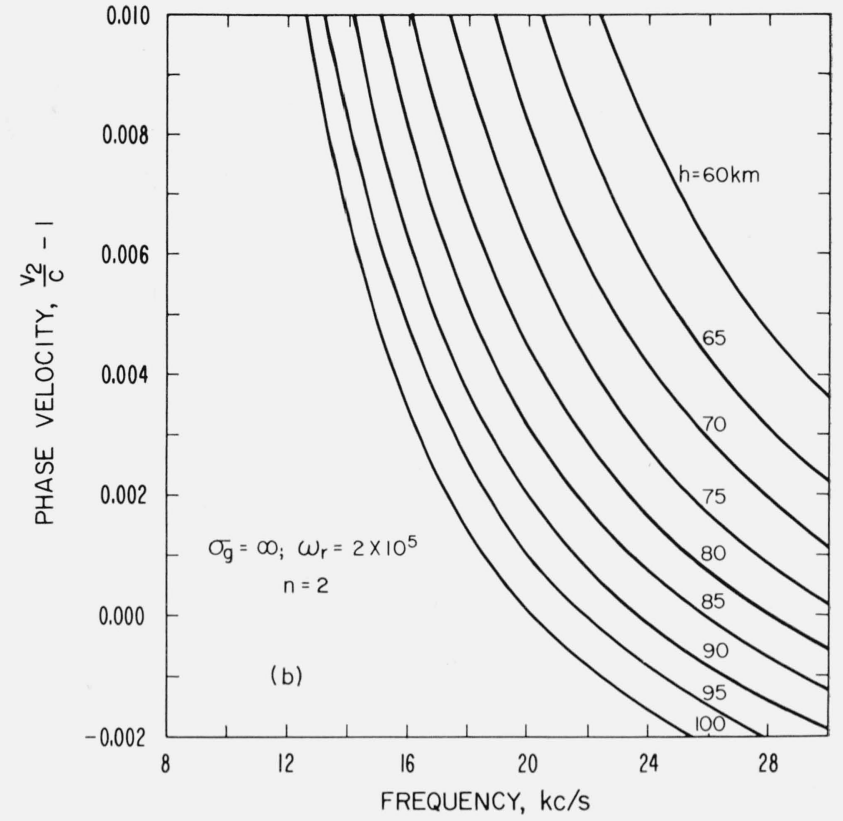

FIGURE 2b. Phase velocity of the second mode for a perfectly conducting earth and a sharply bounded ionosphere.

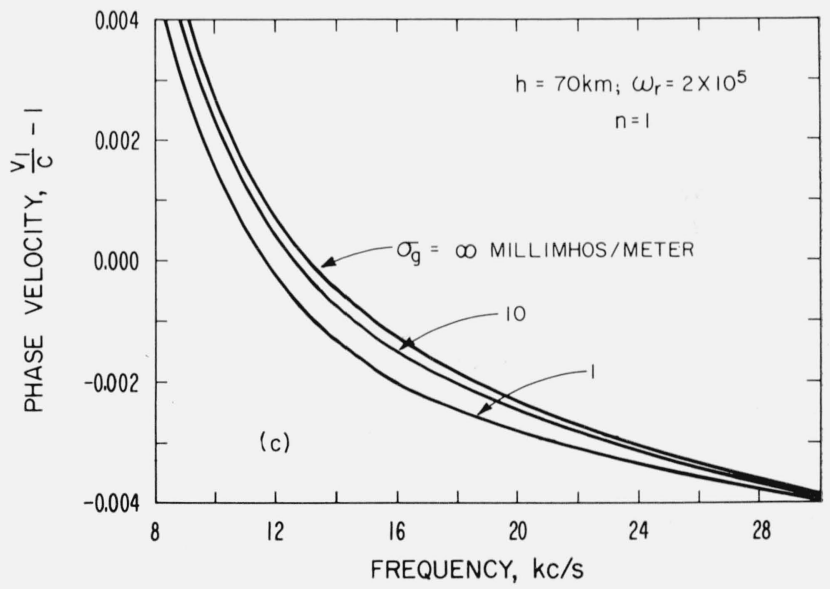

Figure 2c. Phase velocity of the first mode for an imperfectly conducting earth and a sharply bounded ionosphere.

phase velocities differ only slightly from $c$, the velocity of light in vacuo. Nevertheless, this difference is very significant. It is interesting to note that the phase velocity $v_{1}$ of the first mode may be actually equal to $c$ for frequencies around $15 \mathrm{kc} / \mathrm{s}$ when the reflecting heights are around $65 \mathrm{~km}$. For frequencies above this, the phase velocity is less than $c$ and the mode becomes a "slow wave." As indicated by figure $2 \mathrm{c}$, the ground conductivity appears to have only a very small influence on the phase velocity of VLF radio waves. 


\section{A Simplified Form of the Modal Equation}

For certain extensions and other applications, it is important to ascertain if any simplifications can be made to (2). The key step is to recognize that when $t$ is near zero, the factor $i \log \left[w_{2}^{\prime}(t) / w_{1}^{\prime}(t)\right]$ may be replaced by $\pi / 3$. This is valid when $\mid(k a \mid$ $2)^{\frac{1}{3}} C \mid$ is small compared with unity. At the same time, terms such as $\left(C^{2}+2 h / a\right)^{\frac{1}{2}}$ are replaced by

$$
(2 h / a)^{\frac{1}{2}}\left(1+\frac{C^{2} a}{4 h}\right)
$$

which is justified when $C^{2}$ is somewhat less than $h / a$. Employing these approximations, the modal equation (2) reduces to a simple algebraic equation in $C^{2}$. Explicitly, the solution may be written

$$
C_{n}^{2}=\frac{[12 n-5](\pi / 6)-(2 k a / 3)(2 h / a)^{3 / 2}-i \alpha(2 h / a)^{\frac{1}{2}}}{k a(2 h / a)^{\frac{1}{2}}+(i \alpha / 2)(2 h / a)^{-\frac{1}{2}}} .
$$

To give some idea of the goodness of this simple formula, the phase velocity $v_{1}$ for the first mode is shown plotted in figure 3 for $\alpha=0$. It is quite evident, that for most purposes, the approximate method of solution is adequate.

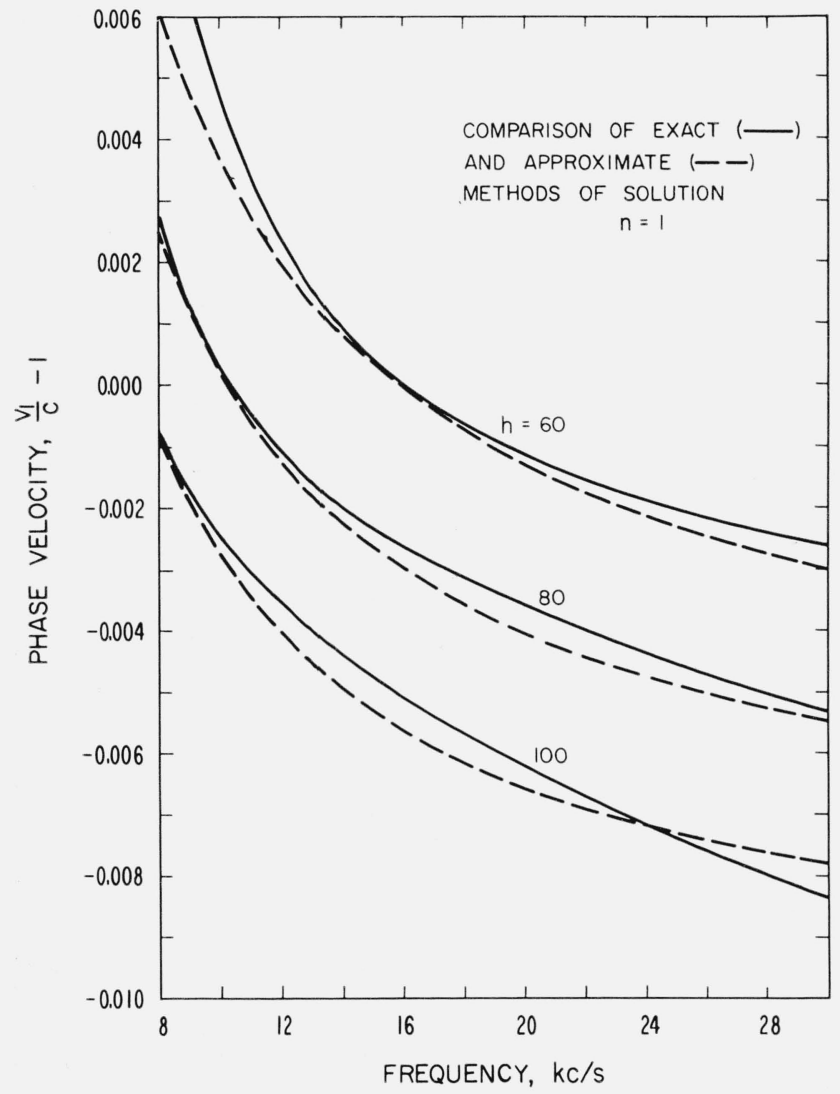

Figure 3. Comparison between accurate or "exact" modal and an approximate form used for simplified calculations.

\section{Exponential Layer}

To illustrate the influence of a non-sharp boundary at the lower edge of the ionosphere, it is assumed that the parameter $\omega_{r}$ varies exponentially with height $z$. Thus

$$
\omega_{r}=\hat{\omega}_{r} \exp (\beta(z-h)),
$$

where $\hat{\omega}_{r}$ is the value of $\omega_{r}$ at the reference height $z=h$. For a typical $D$-layer profile, the exponential form is a surprisingly good fit if $\beta$ is about 0.5 $\mathrm{km}^{-1}$. For daytime, $h$ is approximately $70 \mathrm{~km}$ while, for nighttime conditions, $h$ is about $90 \mathrm{~km}$. For these calculations $\hat{\omega}_{r}$ is assigned the value $2.5 \times 10^{5}$.

The reflection coefficient for such an exponential layer may also be expressed in the form-exp $\left(\alpha C^{\prime}\right)$ when referred to the reference height $z=h$. Furthermore, $\alpha$ is again nearly a constant for a wide range of angles of incidence. A numerical treatment of the exponential layer for plane wave incidence has already been given in some detail [Wait and Walters, 1963]. For present purposes, the complex values of $\alpha$ previously obtained are used in the simplified modal equation (6) given above.

For presentation of results, the attenuation and phase velocity are presented as differences to the corresponding results for the sharply bounded model. This technique has two advantages: (1) it makes use of the more accurate attenuation and phase data using the sharply bounded model, and (2) it illustrates the influence of a non-sharp boundary in a direct manner.

The attenuation increment $\Delta A_{n}$, which is plotted in figures $4 \mathrm{a}, \mathrm{b}, \mathbf{c}$, and $\mathrm{d}$, is defined by

$$
\Delta A_{n}=A_{n}^{(\beta)}-A_{n},
$$

where $A_{n}{ }^{(\beta)}$ is the resultant attenuation rate for the $n$th mode when the ionosphere has an exponential profile, while $A_{n}$ is the attenuation rate of the sharply bounded model with $\omega_{r}=2 \times 10^{5}$.

The attenuation increment $\Delta A_{1}$ for the first mode is shown in figures $4 \mathrm{a}$ and $\mathrm{b}$ for $h$ values of 70 and $90 \mathrm{~km}$. The corresponding attenuation increments $\Delta A_{2}$ for mode 2 are shown in figures $4 \mathrm{c}$ and $\mathrm{d}$. It is interesting to observe that, for the larger values of $\beta$ corresponding to a relatively abrupt boundary, $\Delta A_{n}$ is relatively small and does not depend significantly on frequency. For smaller values of $\beta$, corresponding to a diffuse boundary, the attenuation rate may be significantly increased. This is particularly apparent at the higher frequencies.

The phase velocity increment $N$ is defined in a similar fashion. Explicitly,

$$
N_{n}=\frac{v_{n}^{(\beta)}}{c}-\frac{v_{n}}{c},
$$

where $v_{n}^{(\beta)}$ is the phase velocity of the $n$th mode for an exponential ionosphere while $v_{n}$ is the corresponding phase velocity for the sharply bounded 

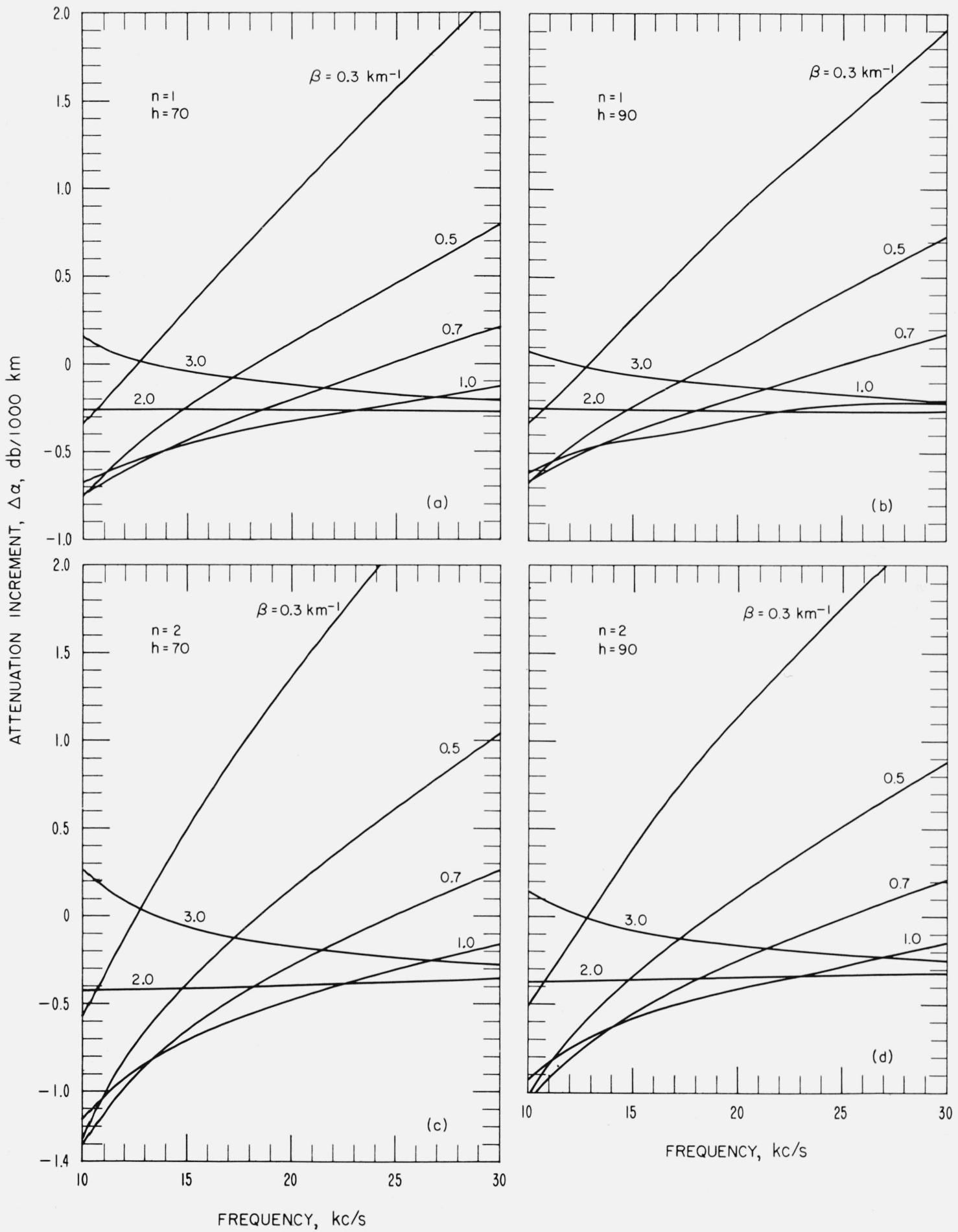

Figures 4a, b, c, and d. The increase or increment of the attenuation for a diffuse ionosphere over that of a sharply bounded ionosphere.

(The effective conductivity of the diffuse ionosphere varies according to exp $[\boldsymbol{\beta}(z-h)]$.) 

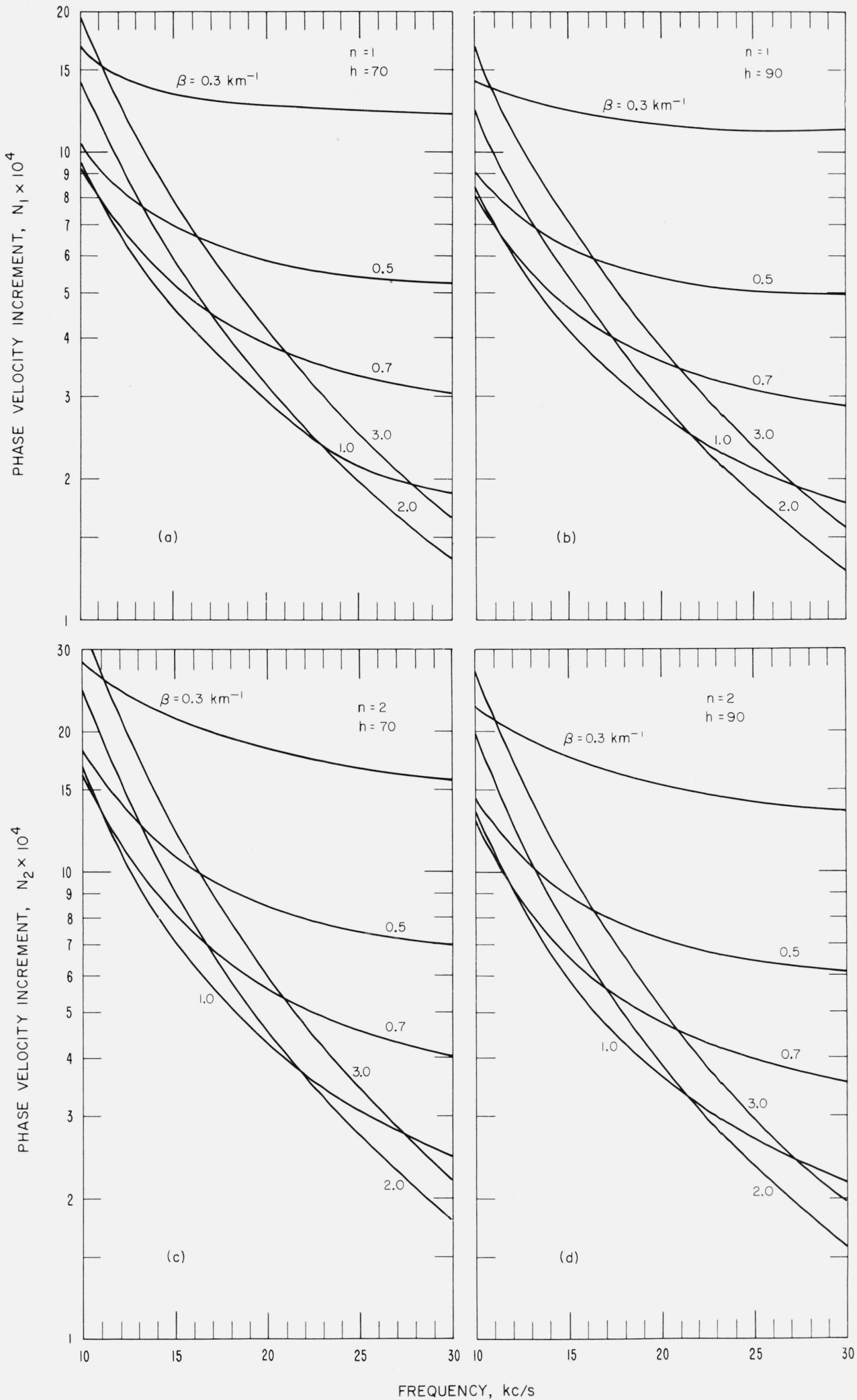

Figures 5a, b, c, and d. The increase or increment of the phase velocity for a diffuse ionosphere over that of a sharply bounded ionosphere. 
model as indicated in figure 5. It is apparent that $N_{n}$ is a small quantity for the range of frequencies indicated. In general, it may be observed that a diffuse ionosphere (i.e., small $\beta$ values) is associated with higher values of phase velocity. Physically, this is a consequence of the lowering of the effective height of reflection. It may be noted that the effect is particularly noticeable for the higher range of (VLF) frequencies.

The actual ionosphere is expected to encompass the whole range of $\beta$ values indicated in figures 4 and 5. Thus, the phase perturbations are a sensitive indicator of the relative diffuseness of the lower ionosphere.

The reader should be cautioned that the results in the present paper make no allowance for the terrestrial magnetic field. This factor will change the attenuation rates to some extent but the conclusions regarding the gradualness of the lower ionosphere are still valid.
The author thanks K. P. Spies and Mrs. L. C. Walters for carrying out the original calculations. The work was supported by the Advanced Research Projects Agency, Washington, D.C., on ARPA Order No. 183-62.

\section{References}

Wait, J. R. (1962), Electromagnetic waves in stratified media (Pergamon Press, Oxford).

Wait, J. R., and L. C. Walters (May-June 1963), Reflection of VLF radio waves from an inhomogeneous ionosphere, Pt. I, J. Research NBS 67D (Radio Prop.), No. 3, 361.

\section{Additional Reference}

Galejs, J. (1963), Terrestrial ELF propagation in the presence of an isotropic ionosphere with an exponential conductivity-height profile, Proceedings of the International Conference on the Ionosphere (Chapman and Hall, Ltd.).

(Paper 67D4-271) 